\title{
Design and Development of Sustained Release Microspheres of Ibuprofen by Emulsification Solvent Evaporation Method Using Polymeric Blend
}

\author{
Nandini Saha', Ikramul Hasan', Mehrina Nazmi ${ }^{1}$ and Md. Selim Reza² \\ ${ }^{1}$ Department of Pharmacy, University of Asia Pacific, Dhanmondi, Dhaka-1209, Bangladesh \\ ${ }^{2}$ Department of Pharmaceutical Technology, Faculty of Pharmacy, University of Dhaka, Dhaka-1000, Bangladesh.
}

\begin{abstract}
Ibuprofen, a non-steroidal anti-inflammatory drug was formulated as microspheres by using Methocel K4M \& Eudragit RSPO. These microspheres were prepared by emulsification solvent evaporation method to provide sustained action and to minimize local side effect of Ibuprofen by avoiding the drug release in the upper gastrointestinal tract. The prepared microspheres were subjected to various evaluation and in-vitro release studies. In-vitro drug release was studied in a paddle type dissolution apparatus (USP Type II Dissolution Apparatus) using Phosphate buffer ( $\mathrm{pH} 7.4$ ) as the dissolution medium at $37.5^{\circ} \mathrm{C}$ for 6 hours (paddle speed $50 \mathrm{RPM}$ ). The release mechanisms were explored and explained with Zero Order, First Order, Higuchi and Korsmeyer-Peppas equations. The correlation coefficients values of the trend lines of the graphs showed that the formulations best fit with Korsmeyer-Peppas release pattern. Microspheres' morphology and chemical integrity were studied by a scanning electron microscope (SEM) and Fourier transforms infrared spectroscopy (FTIR) respectively.
\end{abstract}

Key words: Eudragit RSPO, Methocel K4M, Ibuprofen, Microsphere, Emulsification-Solvent Evaporation method.

\section{Introduction}

Microspheres are one of the multiparticulate drug delivery systems prepared to obtain prolonged and controlled drug delivery, to improve bioavailability or stability and to target specific sites. Microspheres can be defined as solid, approximately spherical particles ranging from 1 to $1000 \mu \mathrm{m}$, containing dispersed drug in either as solution or as microcrystalline form (Wan et al., 1995; Yamuda et al., 2001).

Ibuprofen is a non-steroidal anti-inflammatory drug, which possesses analgesic and mild antipyretic action. Because of its short half life (1-3 hours) it was selected as a model drug in this study. Its activity is more than indomethacin, naproxen and other NSAIDs. Ibuprofen inhibits the inflammation by acting on cyclooxygenase. It also inhibits the lipoxygenase pathway leading to decrease in the production of leukotrienes by the leukocytes and the synovial cells. It also masks $\mathrm{T}$ cell suppressing the production of rheumatoid factors. Most frequent adverse effects occurring with ibuprofen are gastro intestinal disturbance; peptic ulceration, gastrointestinal bleeding have been reported. Sustained drug delivery of ibuprofen will reduce these toxicities considerably by maintaining a low and constant level of drug in the blood (Surendiran and Yuvaraj, 2010).

Microencapsulation is widely used in the pharmaceutical and other sciences to mask tastes or odors, prolong release, impart stability to drug molecules, improve bioavailability, and as multi-particulate dosage forms to produce controlled or targeted drug delivery. It is therefore a rapidly expanding technology for achieving sustained-release dosage forms. These sustained release microspheres may be produced by several methods utilizing emulsion system (oil-in-water, oil-in-oil, waterin-oil-in-water), as well as by Spray drying. The common emulsion system used oil-in-water (o/w), with microspheres being produced by the emulsion solvent evaporation method. This relatively simple method enables the entrapment of a wide range of hydrophobic drugs (Abbaspour et al., 2008; Bolourtchian et al., 2005)

The main objective of this work was to investigate the possibility of obtaining a sustained release formulation of Ibuprofen microspheres by using Eudragit RSPO and Methocel K4M in various drugs, polymer ratios. The 
various physicochemical characteristics and the in-vitro release rates from these microspheres were thus examined (Wan et al., 1995, Yamuda et al., 2001).

\section{Materials and Methods}

Ibuprofen a gift sample from ACI Pharmaceutical limited, Bangladesh, Methocel K4M (Colorcon, USA), Eudragit RSPO (EVONIK, Germany), Dichloromethane (DCM, Merck, Germany), Ethanol (Merck, Germany), Light liquid paraffin (Merck, Germany), $n$-hexane (Merck, Germany), Span 80 (Merck, Germany) were purchased from local market. All other ingredients were of analytical grade.

Preparation of ibuprofen microspheres: The oil in oil $(\mathrm{O} / \mathrm{O})$ type of emulsion formation technique was adopted as the ideal technique for the study to avoid the heating which is necessary in case of Oil in Water type emulsion. Calculated quantity of polymer was dissolved in 1:1 ratio of ethanol and dichloromethane which acts as internal phase shown in table 1 to form a homogenous polymer solution. Light liquid paraffin was added up to $100 \mathrm{ml}$ along with $1 \%$ span 80 and stirring was started at 1000 rpm and continued for 5 minutes. Then required amount of Ibuprofen powder respective to the batch was added with external phase drop wise and stirred for $3 \mathrm{hrs}$ at $1000 \mathrm{rpm}$. Prepared microspheres were washed with n-hexane repeatedly and allowed to dry at room temperature over night to get free flowing microspheres. By varying this drug polymer ratio, six batches of microspheres were prepared (Table 1).

Table 1. Formulation design of all the formulation.

\begin{tabular}{lcccccc}
\hline Ingredients & F1 & F2 & F3 & F4 & F5 & F6 \\
\hline Drug (gm) & 1 & 1 & 1 & 1 & 1 & 1 \\
Methocel K4M (gm) & 0.25 & 0.50 & 1.0 & 1.5 & 2.0 & 2.5 \\
Eudragit RSPO (gm) & 0.25 & 0.50 & 1.0 & 1.5 & 2.0 & 2.5 \\
Ethanol (ml) & 5 & 5 & 5 & 5 & 5 & 5 \\
DCM (ml) & 5 & 5 & 5 & $1 \%$ & 5 & 5 \\
Span 80 & $1 \%$ & $1 \%$ & $1 \%$ & & $1 \%$ \\
Paraffin oil (ml) & & \multicolumn{2}{c}{ Up to $100 \mathrm{ml}$} & \\
\hline
\end{tabular}

Incorporation efficiency: Incorporation efficiency was determined for all batches using the following formulas. Values are expressed as percentage.

Drug incorporation efficiency $=\left(\frac{M_{\text {actual }}}{M_{\text {theoretical }}} \times 100\right) \%$

In vitro dissolution study of microspheres containing Ibuprofen: In vitro dissolution study was performed in a paddle type (Type II) Dissolution Apparatus. $100 \mathrm{mg}$ equivalent of microspheres were taken from each batch of formulation for dissolution purpose. Phosphate buffer of $\mathrm{pH} 7.4$ was used as dissolution media, paddle speed was set at $50 \mathrm{rpm}$ and temperature was maintained fixed at $37.5^{\circ} \mathrm{C}$. The fixed amount of microsphere from each batch was weighed and transfer in each dissolution basket. The dissolution process was carried out for 6 hours and 10 $\mathrm{ml}$ dissolution sample from each dissolution media was withdrawn at a predetermined intervals of 15 minutes, 30 minutes, 1 hour, 2 hour, 3 hour, 4 hour, 5 hour, 6 hour.
Each and every time $10 \mathrm{~m} 1$ of dissolution sample was compensated by fresh $10 \mathrm{ml}$ phosphate buffer (pH 7.4).

Shape and surface characterization of microspheres by scanning electron microscopy (SEM): The optimized microspheres were taken for the shape and surface characteristic studies. The microspheres from the optimized batch were mounted on the SEM sample stab (aluminium stabs) which were coated with a double-sided sticking tape, sealed and finally coated with gold $\left(200 \mathrm{~A}^{\circ}\right)$ under reduced pressure ( 0.001 torr) for 15 minutes using an Ion sputtering device. The gold coated samples were scanned using scanning electron microscope (S-3400N, Hitachi) under varying magnifications and photomicrographs of suitable magnifications were obtained. The microspheres were dried completely before examination (Chuanbin et al., 2001).

Fourier transform infrared spectroscopy (FTIR) study: Drug-polymer interactions were studied by FTIR spectroscopy. The spectra were recorded for pure drug and 
drug-loaded microspheres using FTIR. Samples were prepared in $\mathrm{KBr}$ disks (2 mg sample in $200 \mathrm{mg} \mathrm{KBr}$ ). The mixtures got were taken in a diffuse reflectance sampler and IR spectra recorded by scanning in the wavelength region of 400 to $4000 \mathrm{~cm}^{-1}$ in a FTIR Spectrophotometer (model 460 Plus, Jasco, Japan).

\section{Results and Discussion}

Incorporation efficiency: Drug incorporation efficiency has linear relationship with the amount of polymer used. Incorporation efficiency had increased with increase in the percentage of polymer. F1 showed lowest efficiency (42\%) which is the formulation of $66 \%$ drug loading where as F6 showed highest efficiency (84.57\%) which is the formulation of $16 \%$ drug loading.

Table 2. Incorporation efficiency (\%) of all the formulations.

\begin{tabular}{cc}
\hline Formulations & Incorporation efficiency (\%) \\
\hline F1 & 42 \\
F2 & 65.2 \\
F3 & 65.97 \\
F4 & 69 \\
F5 & 76.35 \\
F6 & 84.57 \\
\hline
\end{tabular}

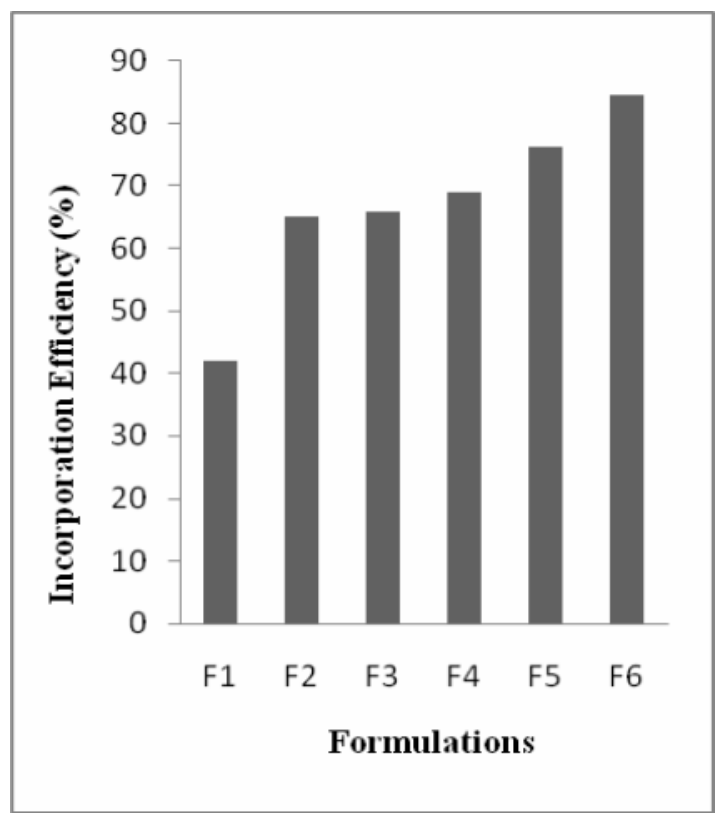

Figure 1. Bar diagram of incorporation efficiency.

Effect of methocel K4M \& eudragit RSPO on release kinetics of ibuprofen from microspheres: For finding the release profile dissolution testing of all formulations (F1 to F6) was carried out in phosphate buffer ( $\mathrm{pH}$ 7.4) for 6 hours at $50 \mathrm{rpm}$ and release rate of Ibuprofen in phosphate buffer was calculated. Different release profile were plotted to find out the best fitted models and to observe the release pattern of Ibuprofen from the Methocel K4M \& Eudragit RSPO microspheres.

From the zero order release profile, it can be observed that the release rate of Ibuprofen were $96.356 \%, 83.12 \%$, $78.405 \%, 72.723 \%, 71.458 \%$, and $68.498 \%$ within 6 hours for F1, F2, F3, F4, F5 \& F6 respectively. Thus the results showed that the release rate of Ibuprofen from the microspheres is decreasing as the ratio of polymer is increasing.

Data treatment of release profiles of ibuprofen from Methocel K4M and Eudragit-RSPO microspheres considering, $R^{2}, n, T_{25 \%}, T_{50 \%}, T_{80 \%}$ and MDT values: From table 3 it is observed that all the formulations F1 to F6 was best fitted to the korsmeyer-peppas equation. The value of $\mathrm{n}$ is within 0.45 to 0.85 and the value of $\mathrm{n}$ was reduced with the decrease of concentration by following Non Fickian release pattern more specifically diffusion controlled release mechanism which means the zero order was changed over time (Higuchi, 1963; Korsmeyer et al., 1983; Peppas, 1985).

The successive fractional dissolution time values are shown in table 4. It can be observed from the values that $\mathrm{T}_{25 \%}, \mathrm{~T}_{50 \%}, \mathrm{~T}_{80 \%}$ and MDT of $\mathrm{F} 1$ to $\mathrm{F} 6$ gradually increase. As the concentration of polymer increases the drug release rate gradually decreases and the values of $\mathrm{T}_{25 \%}, \mathrm{~T}_{50 \%}, \mathrm{~T}_{80 \%}$ and MDT gradually becomes higher.

Surface morphology and particle size distribution study of microspheres by SEM study: SEM study revealed that the microspheres prepared from Methocel K4M and Eudragit RLPO are generally roughly spherical with drug on the surface (Figure A). From Figure $4 \mathrm{~B}$ it is revealed that all the microspheres were within the size range of microsphere $(1-1000 \mu \mathrm{m})$. Particle sizes of about nine microspheres were measured by using scanning electron microscope (Figure 4B). The particle sizes were $574 \mu \mathrm{m}$, $388 \mu \mathrm{m}, 941 \mu \mathrm{m}, 721 \mu \mathrm{m}, 648 \mu \mathrm{m}, 875 \mu \mathrm{m}, 650 \mu \mathrm{m}, 669 \mu \mathrm{m}$ and $543 \mu \mathrm{m}$. So in terms of particle size the microspheres complied with the compendial specification. 

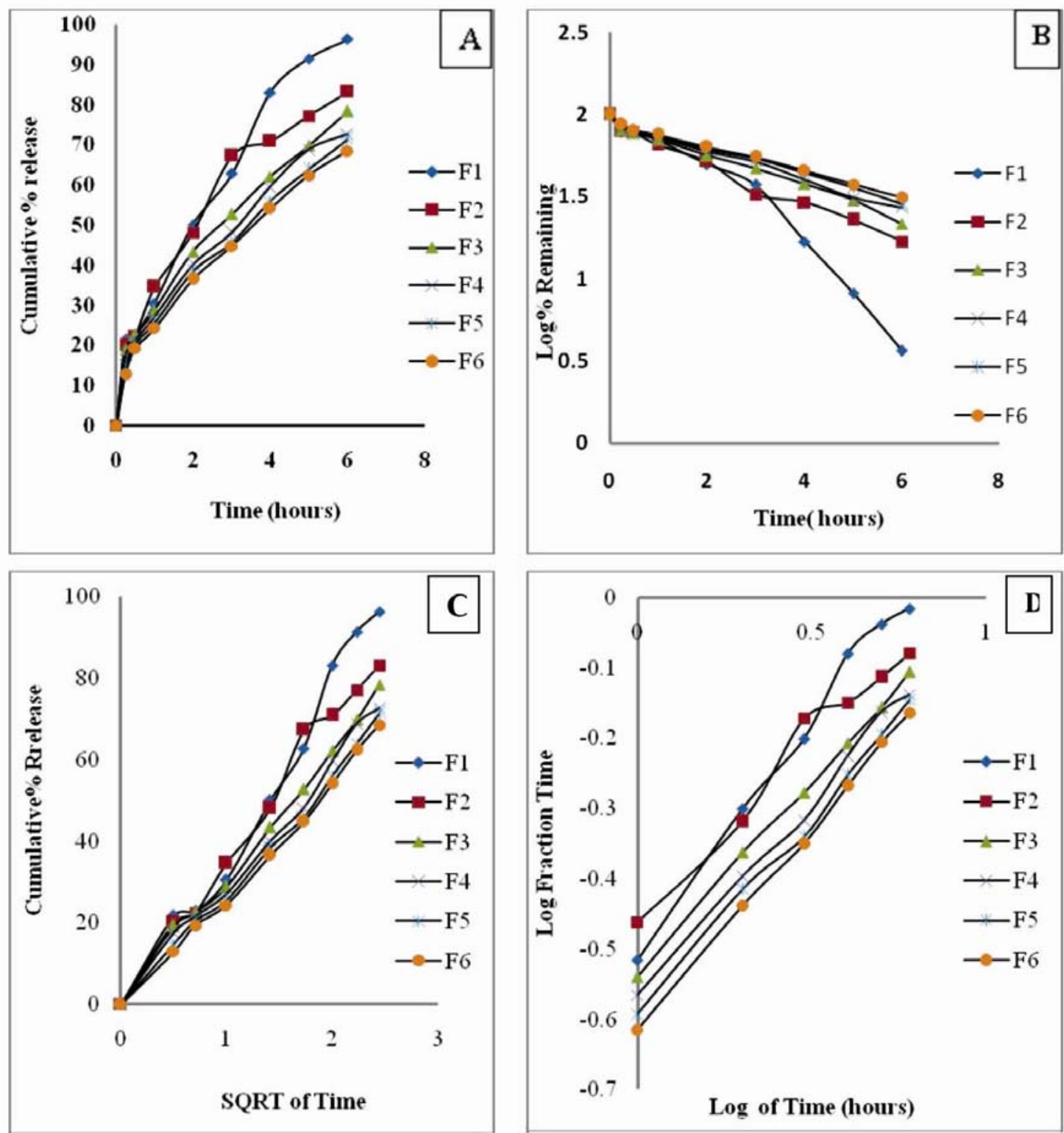

Figure 2. In-vitro Release kinetics of Ibuprofen from Methocel K4M and Eudragit-RSPO microspheres A. zero order plot, B. First order plot, C. Higuchi plot, D. Korsmeyer plot.

Table 3. Interception of release rate constant and R-squared values for different release kinetics of Methocel K4M \& Eudragit RSPO based ibuprofen microspheres.

\begin{tabular}{ccccccccc}
\hline & \multicolumn{2}{c}{ Zero order } & \multicolumn{2}{c}{ First order } & \multicolumn{2}{c}{ Higuchi } & \multicolumn{2}{c}{ Korsmeyer } \\
\cline { 2 - 8 } Formulations & $\mathrm{K}_{0}$ & $\mathrm{R}^{2}$ & $\mathrm{~K}_{1}$ & $\mathrm{R}^{2}$ & $\mathrm{~K}_{\mathrm{H}}$ & $\mathrm{R}^{2}$ & $\mathrm{n}$ & $\mathrm{R}^{2}$ \\
\hline F1 & 0.26 & 0.945 & -0.221 & 0.983 & 40.77 & 0.983 & 0.663 & 0.991 \\
F2 & 0.21 & 0.923 & -0.112 & 0.986 & 34.77 & 0.99 & 0.501 & 0.996 \\
F3 & 0.192 & 0.943 & -0.099 & 0.988 & 31.02 & 0.995 & 0.549 & 0.999 \\
F4 & 0.183 & 0.945 & -0.089 & 0.99 & 29.54 & 0.993 & 0.56 & 0.995 \\
F5 & 0.177 & 0.953 & -0.082 & 0.989 & 28.5 & 0.994 & 0.567 & 0.996 \\
F6 & 0.173 & 0.956 & -0.077 & 0.992 & 27.84 & 0.995 & 0.579 & 0.998 \\
\hline
\end{tabular}


Table 4. Successive fractional dissolution times (hours) of F1 to F6.

\begin{tabular}{ccccc}
\hline \multirow{2}{*}{ Formulations } & \multicolumn{4}{c}{ Successive fractional dissolution time } \\
& \multicolumn{4}{c}{ (hrs) } \\
\cline { 2 - 5 } & $\mathrm{T}_{25 \%}$ & $\mathrm{~T}_{50 \%}$ & MDT & $\mathrm{T}_{80 \%}$ \\
\hline F1 & 0.901 & 2.254 & 2.428 & 4.199 \\
F2 & 0.506 & 2.022 & 2.696 & 5.178 \\
F3 & 0.758 & 2.679 & 3.356 & 6.307 \\
F4 & 0.876 & 3.022 & 3.740 & 6.995 \\
F5 & 0.964 & 3.272 & 4.021 & 7.498 \\
F6 & 1.053 & 3.485 & 4.231 & 7.849 \\
\hline
\end{tabular}

Fourier transform infrared spectrometry (FTIR): FTIR was done to examine drug-polymer interaction. According to the figure 6, it shows the presence of C-N group, $\mathrm{N}=\mathrm{O}$ group, $\mathrm{C}-\mathrm{H}$ group and $\mathrm{C}-\mathrm{C}$ group at 1069.71 $\mathrm{cm}^{-1}, 1326 \mathrm{~cm}^{-1}, 2895 \mathrm{~cm}^{-1}$ and $1227 \mathrm{~cm}^{-1}$, respectively. These peaks are identical for pure ibuprofen. These peaks were found unchanged in figure 5 which is the FTIR of F4. This proves that there were no drug-polymer interactions

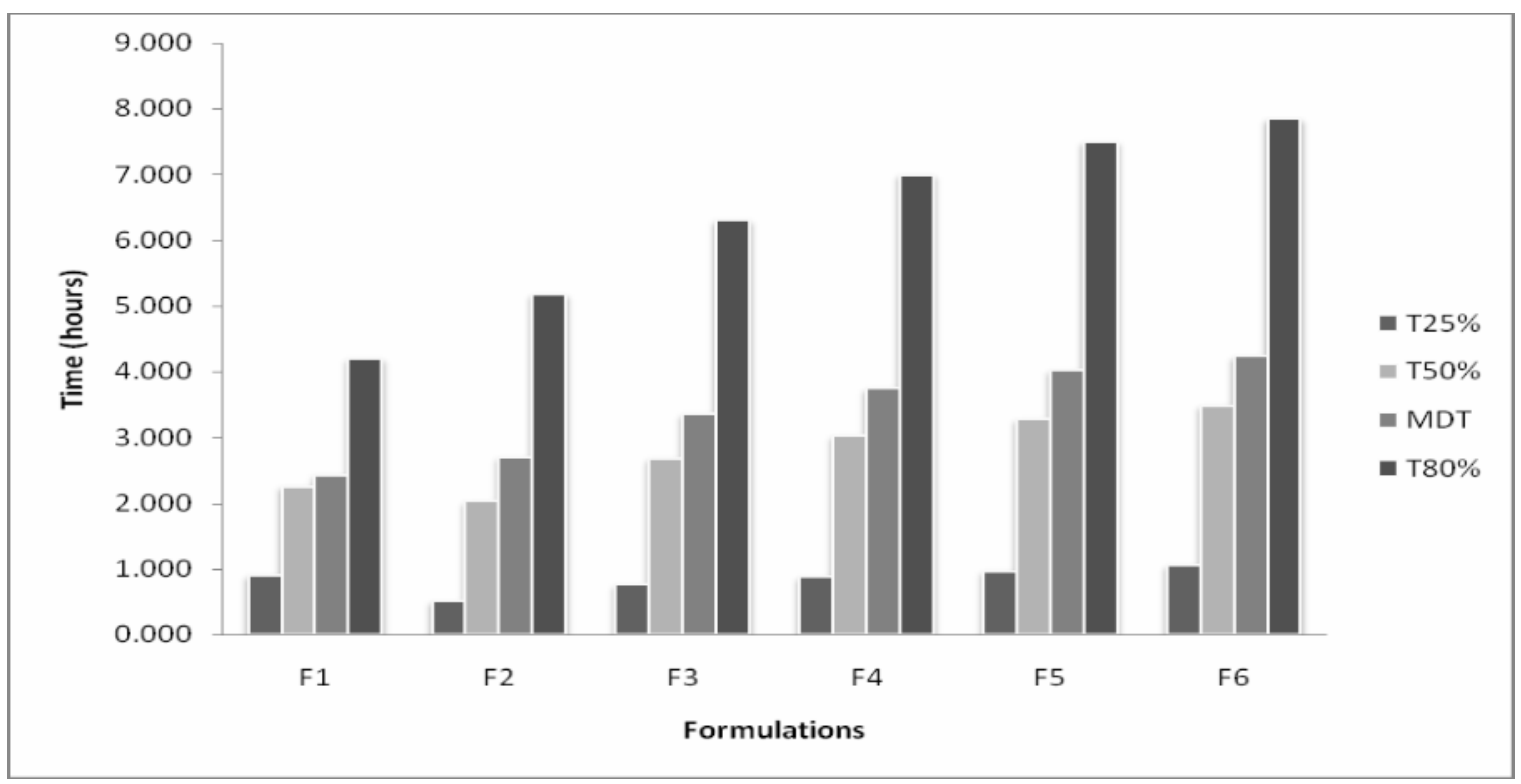

Figure 3. Successive fractional dissolution times (hours) of ibuprofen from Methocel K4M \& Eudragit RSPO microspheres F1 to F6.
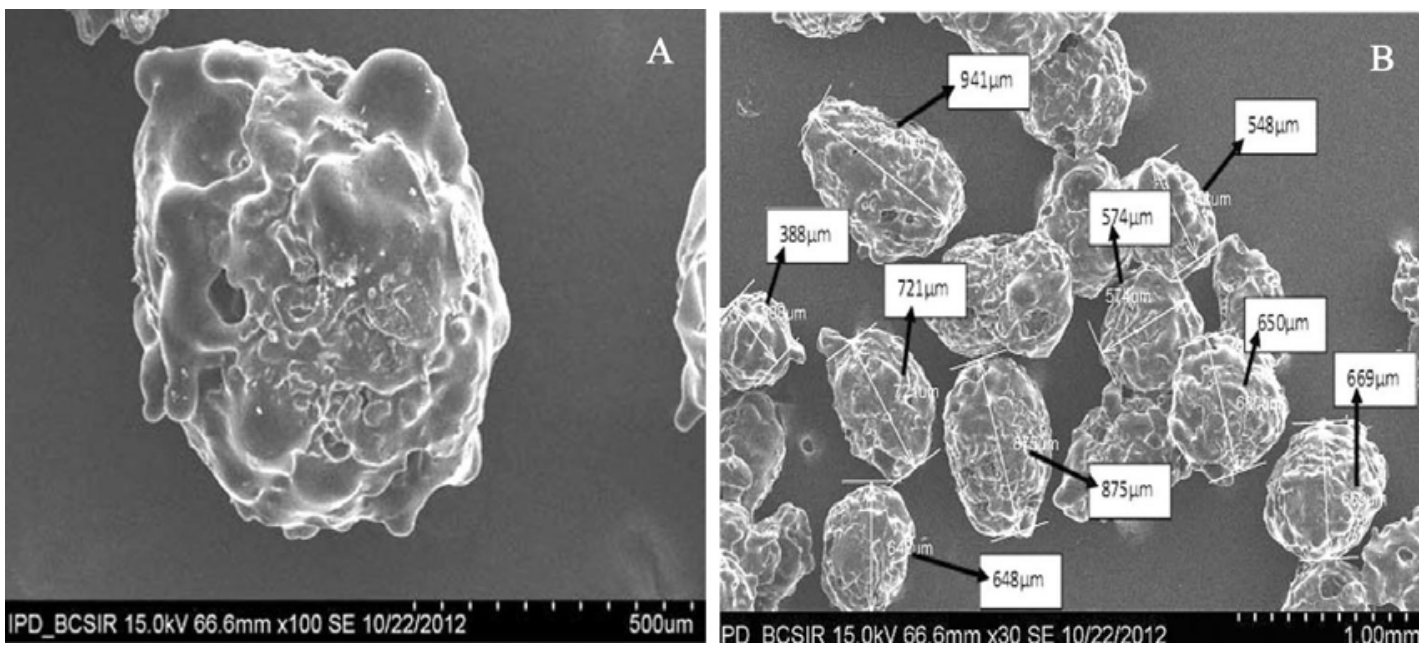

Figure 4 (A and B). Scanning electron microscopic study of microspheres. 


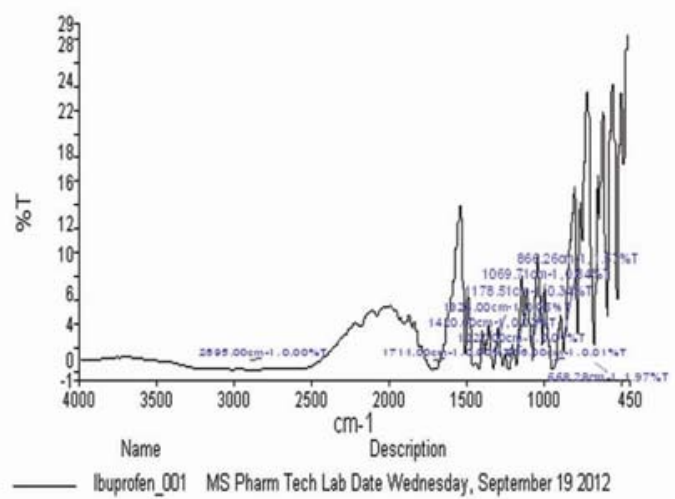

Figure 5. FTIR of pure ibuprofen.

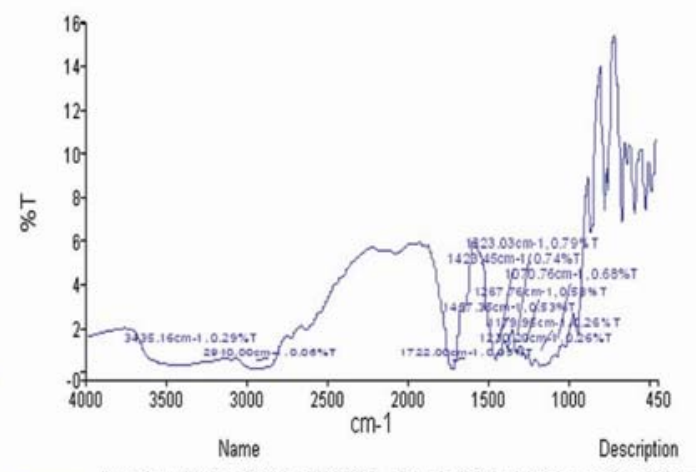

lbuprofen + Methocel K4M + EU RSPO Sample 023 By Administrator Date Wednesday,

Figure 6. FTIR of microspheres.

Higuchi, T. 1963. Mechanism of sustained action medication, theoretical analysis of rate of release of solid drugs dispersed in solid matrices. J. Pharm. Sci. 52, 1145-1149.

Horoz, B., Kiliic, M., Arslan, N. and Baykara, Y.T. 2004. Effect of different dispersing agents on the characteristics of Eudragit microspheres prepared by a solvent evaporation method. $J$. Microencap. 21, 191-202.

Korsmeyer, R.W., Gurny, R., Doelker, E.M., Buri, P. and Peppas, N.A. 1983. Mechanism of solute release from porous hydrophilic polymers. Int. J. Pharm. 15, 25-35.

Li, M., Rouaud, O. and Ponecelet, D. 2008. Microencapsulation by Solvent Evaporation: State of the art for process engineering approaches. Int. J. of Pharm. 363, 26-29.

Peppas, N.A. 1985. Analysis of Fickian and non-Fickian drug release from polymers. Pharm. Acta. Helv. 60, 110-111.

Sethi, R.K., Guru, P., Sahoo, S.K., Das, P.K. and Barik, B.B. 2010. Design and development of drug Loaded Microsphere by utilizing Emulsion Solvent Evaporation Method. Int. J. of Pharm. Sci. 1, 4649.

Surendiran, N.S. and Yuvaraj, T.V. 2010. Preparation and evaluation of ibuprofen microspheres by using co-acervation phase separation technique. Int. J. Chem. Tech. Res. 2, 1214-1219.

Wan, L.S. and Chui, W.K. 1995. Deviation of the ratio of drugs in a two component mixture with ethyl cellulose and carboxymethyl ethyl cellulose. J. Microencapsul. 12, 417-23.

Yamuda, T., Onishi, H. and Machida, Y.2001. Sustained release ketoprofen microparticles with ethyl cellulose and carboxymethyl ethyl cellulose. J. Controlled Release. 75, 271-282.

Zaghloul, A.A., Faltinek, J., Vaithiyalingam, S.R., Reddy, I.K. and Khan, M.A. 2001. Screening of process and formulation variables for the preparation of extended release microspheres. Die Pharmazie. 56, 321-324.

Zhu, K.J., Li, Y., Jiang, H.L., Yasuda, H., Ichimaru, A., Yamamoto, K., Lecomte, P. and Jerome, R. 2005. Preparetion, characterization and in vitro release properties of ibuprofen loaded nicrospheres based on polylactide, poly ( $\alpha$-caprolactone) and their copolymers. $J$. Microencap. 22, 25-36. 